\title{
EI compromiso como variable mediadora para la predicción de las futuras intenciones de consumo en los servicios. Una aproximación empírica a los consumidores de artes escénicas en España ${ }^{1}$
}

\section{Commitment as a Mediator Variable Used to Predict Future Service Consumption Intentions: An Empirical Approach to Consumers of Performing Arts in Spain}

\author{
María José Quero Gervilla ${ }^{2}$ \\ Rafael Ventura Fernández ${ }^{2}$ \\ Universidad de Málaga
}

Recibido el 11 de junio de 2009, aceptado el 8 de marzo de 2010

$\mathrm{N}^{\mathrm{o}}$ de clasificación JEL: M3

DOI: $10.5295 / \mathrm{cdg} .100163 \mathrm{mq}$

Reseña bibliográfica: QUERO, M. J. y VENTURA, R. (2011): "El compromiso como variable mediadora para la predicción de las futuras intenciones de consumo en los servicios. Una aproximación empírica a los consumidores de artes escénicas en España”, Cuadernos de Gestión, Vol 11, $\mathrm{n}^{\circ} 1$, pp.15-36, DOI: $10.5295 / \mathrm{cdg} .100163 \mathrm{mq}$

\section{Resumen:}

El presente trabajo de investigación trata de arrojar luz sobre las relaciones entre las variables Satisfacción, Compromiso, Confianza y Futuras Intenciones de compra. Con este fin, se propone un Modelo de Gestión de las Relaciones con Clientes de Servicios en el que se observa que la variable más importante en la consecución de resultados positivos en lo que respecta a intenciones de asistencia futura de los consumidores es el Compromiso. El trabajo empírico se desarrolla en el sector servicios, concretamente en el ámbito de la exhibición de artes escénicas. Con este fin se han entrevistado personalmente a 1005 consumidores de artes escénicas en la entrada y salida de los espectáculos. En él han colaborado 150 teatros públicos y privados de toda España.

\footnotetext{
${ }^{1}$ Trabajo realizado en el marco del proyecto de investigación "El enfoque de marketing relacional en el mercado de las artes escénicas: análisis y propuestas de actuación", financiado por la Red Española de Teatros, Auditorios y Circuitos de Titularidad Pública y la Universidad de Málaga a través del grupo de investigación SEJ314.

${ }^{2}$ Departamento de Economía y Administración de Empresas, Área de Comercialización e Investigación de Mercados. Facultad de Ciencias Económicas y Empresariales. Universidad de Málaga. Campus El Ejido nº 6, 29071 Málaga. E-mail:mqg@uma.es; rventura@uma.es
} 
El compromiso como variable mediadora para la predicción de las futuras intenciones de consumo en los servicios...

\title{
Palabras clave:
}

Marketing relacional, satisfacción, compromiso, confianza, futuras intenciones.

\begin{abstract}
:
The aim of this research project is to gain some insight into the relationships between the following variables: Satisfaction, Commitment, Trust, and Future Purchasing Intentions. In order to achieve this aim, a Model for the Management of Service Sector Customer Relations is proposed, and this Model shows that the most important variable for achieving positive results in terms of future attendance intentions amongst consumers is Commitment. This empirical work focuses on the services sector, specifically on the field of performing arts. In order to carry out the analyses, 1005 performing art consumers were interviewed at the entrances or exits to shows. 150 public and private theatres from throughout the whole of Spain have collaborated in the project.
\end{abstract}

\section{Key Words:}

Relational marketing, satisfaction, commitment, trust, future intentions. 


\section{INTRODUCCIÓN}

¿Existe alguna evidencia de que niveles altos de compromiso en el consumidor se traduzcan en un incremento de consumo o de intenciones de consumo? ¿Están justificadas las teorías de marketing relacional que tienen como objetivo incrementar la satisfacción, la confianza y el compromiso en el cliente? La literatura de marketing relacional ha tratado ampliamente la importancia de estas variables en la construcción de relaciones con el cliente, sin embargo son pocos los trabajos dirigidos a identificar los resultados que se derivan de una adecuada gestión de estas variables.

Las relaciones con el cliente son el eje central en el desarrollo de las estrategias de marketing relacional y generan múltiples beneficios para las organizaciones (Garbarino y Jonson, 1999; Henning-Thurau et al., 2002; San Martin et al., 2004; Christopher, et al., 2007) pero sería conveniente conocer cómo se relacionan estas tres variables entre sí y qué beneficios concretos pueden ofrecer a la organización con el fin de poder diseñar las políticas de marketing de una manera más eficiente. En el presente trabajo de investigación proponemos un modelo que relaciona las variables satisfacción, compromiso y confianza con las intenciones futuras de consumo. El trabajo empírico se desarrolla en el sector servicios, concretamente en el ámbito de la exhibición de artes escénicas. Con este fin se han entrevistado personalmente a 1005 consumidores de artes escénicas a la entrada y salida de los espacios. En él han colaborado 150 teatros públicos y privados de toda España.

El Modelo de Gestión de las Relaciones con Clientes de Servicios propuesto demuestra que la variable más importante en la consecución de resultados positivos en lo que respecta a intenciones de asistencia futura de los consumidores es el compromiso. La satisfacción y la confianza son importantes en la medida en que sirven para construir el compromiso del cliente con la organización, pero altos niveles de satisfacción o de confianza no aseguran que el cliente incremente sus intenciones de consumo futuro.

Para el desarrollo del Modelo de Gestión de las Relaciones con Clientes de Servicios hemos utilizado como base dos modelos: El modelo de Compromiso - Confinaza desarrollado por Morgan y Hunt (1994) y ampliamente analizado en la literatura de marketing de relaciones y los dos modelos desarrollados por Garbarino y Johnson (1999) para identificar las diferencias en la estructura de relaciones entre variables dependiendo del nivel de compromiso de los consumidores, dando lugar a dos modelos: Modelo de Compromiso y Confianza como Mediadores, para consumidores con un alto nivel de relación con la organización, y Modelo de Satisfacción Global Mediadora, para consumidores que desarrollan una transacción de tipo relacional con la organización. La clasificación de los clientes en grupos de vinculación alta o baja se mide por su condición de abonados o no abonados, criterio que también ha sido utilizado en el entorno cultural por Voss y Voss (2000). Este mismo criterio ha sido utilizado en nuestro trabajo de investigación, con la peculiaridad de que nos hemos centrado exclusivamente en los consumidores no abonados con el fin de analizar otros valores de compromiso existente entre los consumidores y distinto del vínculo estrictamente contractual. Además, el mercado español de servicios culturales se caracteriza por la existencia de un porcentaje muy bajo de abonados, lo que hace aún más importante el análisis de vínculos de compromiso no contractual, como es la actitud del consumidor. 
La finalidad de este estudio es aportar información sobre las variables que inciden en ese proceso de vinculación del cliente con la organización que va desde un intercambio transaccional hasta un intercambio de socio (relacional), en el que el cliente, más allá de consumir el producto, se ve identificado con la marca, con la organización, le preocupa lo que pueda pasarle y difunde información positiva sobre ella. Para una organización de servicios, resulta altamente deseable que el máximo nivel de consumidores alcancen el nivel de socios o clientes fieles, ya que es el único capaz de asegurar una intención futura de consumo, además de generar las múltiples sinergias positivas investigadas por la literatura de marketing relacional.

Aunque hay muchos trabajos empíricos que relacionan las variables Satisfacción, Compromiso y Confianza de forma individual, son pocos los que han tratado de aportar información en el conocimiento de la relación entre ellas. Si pretendemos desarrollar estrategias de fidelización de clientes de forma eficiente en el sector servicios, es importante saber qué tipo de resultado conseguimos si incrementamos la satisfacción, o el compromiso o la confianza en la intencionalidad de consumo futuro del cliente.

\section{DEL CRM AL CMR: HACIA UNA INTEGRACIÓN DEL CLIENTE EN LA GESTIÓN DE LAS RELACIONES}

En los últimos años, el mercado ha sufrido cambios que han provocado una alteración profunda en la gestión de las relaciones entre el cliente y la organización. En los años 70 era generalmente aceptado el concepto de marketing como "ciencia de las transacciones”, siendo el núcleo de la transacción el intercambio (Bagozzi, 1975), y en torno a este concepto se viene a construir toda una teoría general de marketing que alcanza su punto álgido con la teoría del Marketing Mix difundida por McCarthy (1960) y posteriormente por Kotler (1967). Esta teoría ha constituido durante años un paradigma que ha servido como marco para el desarrollo de la ciencia del marketing. Sin embargo, este paradigma, de aceptación consensuada entre los estudiosos de esta ciencia, comienza a mostrar limitaciones e insuficiencias en el entorno actual, provocando diversas críticas, así como el desarrollo de modelos alternativos que buscan una mejor adaptación a la realidad actual. Estas deficiencias han provocado que a finales de los años 80 se inicie una línea de pensamiento denominada marketing relacional, en la que han participado de forma muy directa los investigadores del ámbito del marketing de servicios, lo que explica en gran medida las estructuras adoptadas en su desarrollo. Esta perspectiva viene a reconocer que centrarse exclusivamente en la transacción puede llevar a ignorar gran parte de la esencia del concepto de marketing (Houston y Gassemheimer, 1987). En este sentido, la definición de Hunt (1983, p. 13) del marketing como "la ciencia del comportamiento que busca explicar las relaciones de intercambio" muestra cómo comienza a reconocerse un cambio en el objeto principal del marketing que va de la pura transacción a las relaciones. El debate más reciente a este respecto nos lo ofrecen Vargo y Lush (2004, p. 1): "Las nuevas perspectivas están convergiendo hacia la formación de una nueva lógica de marketing, en la cual resulta fundamental para el intercambio el enfoque de los servicios en oposición al de los bienes". No es, por lo tanto, casual que las nuevas teorías de marketing relacional estén siendo desarrolladas por consolidados investigadores del 
marketing de servicios, ello se debe a que estamos asistiendo a un cambio sustancial en el concepto de producto, en el que se borran las barreras entre bienes y servicios para unificar bajo una misma perspectiva su gestión, con la peculiaridad de que esa perspectiva se asemeja en gran medida a la de los servicios, cuyas características diferenciales también comienzan a cuestionarse (Lovelock y Gummesson, 2004).

Este cambio de objetivo ha supuesto, desde la perspectiva de diversos autores (Gummesson, 2008; Christopher et al., 2007; Sheth y Parvatiyar, 2000), un cambio de paradigma. Aunque presentan diferencias sustanciales en su configuración, las diferentes teorías desarrolladas en el campo del marketing relacional coinciden en considerar que el desarrollo de relaciones con los clientes constituye la fuente más importante de relaciones para las organizaciones (Christopher et al., 2007; Bonnemaizon et al., 2007).

Las aportaciones más recientes realizadas en el ámbito del marketing relacional dan un paso adelante en los modelos clásicos de relaciones (Morgan y Hunt, 1994; Christopher et al., 2007), para intentar introducir al cliente en el modelo de gestión. En este sentido, el estudio Delphi desarrollado por Bonnemaizon et al. (2007), en el que participan los investigadores europeos más representativos de cada país con el fin de pronosticar las tendencias en la investigación del marketing relacional, llega a la conclusión que la nueva tendencia llevará a las organizaciones a tratar de conocer las expectativas del cliente, y esta información será la que dirija su gestión (Customer Experience Management), desarrollando un papel más activo y poderoso en la relación, acentuándose la involucración del cliente en el proceso de creación de valor. En esta misma línea, Law et al. (2003) consideran que los clientes han de desempeñar un papel más partcipativo en la organización, de tal manera que las estrategias de relación han de estar parcialmente dirigidas por ellos, surgiendo un nuevo término para la gestión de relaciones con clientes: el CMR (Customer-Managed Relationship, Relación orientada por el cliente) en oposición al tradicional CRM (Customer Relationship Management), en el que el cliente adoptaba una posición más pasiva.

En el ámbito de los servicios culturales estas teorías han de desarrollarse teniendo en cuenta que modelo de marketing general presenta peculiaridades que lo diferencian de la estructura clásica de comercialización (Hirshman, 1983; Hill et al., 2001; Colbert, 2001): En primer lugar, el proceso de marketing empieza en el ente productor, y desde este origen (producto cultural), se ha de decidir la parte del mercado que puede estar interesada en su consumo. En un segundo paso, una vez identificados los consumidores potenciales, la empresa decidirá sobre las restantes estrategias de marketing. Nos enfrentamos, por lo tanto, a un tipo de mercado cuyo proceso de comercialización presenta una estructura del tipo "product - to - client" (Colbert, 2001, p. 16). Esta estructura atípica transforma la política de relaciones con el cliente, al considerarse que el núcleo del servicio es inalterable. Sin embargo, no debemos confundir el núcleo del servicio (entendido como el entretenimiento a través del consumo de un producto cultural) con el servicio global, que incorpora una amplia variedad de servicios de proceso y de apoyo (Lovelock, 1999) que desempeñan un papel clave en el proceso de creación y percepción de valor por parte de la audiencia. Es en este ámbito en el que se ubican las estrategias de CMR como un conjunto de actividades dirigidas a identificar y satisfacer las demandas relacionales de los clientes, incorporándolos activamente al proceso de creación de valor. 


\section{MODIFICACIÓN DEL COMPORTAMIENTO DEL CONSUMIDOR CULTU- RAL EN FUNCIÓN DEL VÍNCULO DESARROLLADO CON LA ENTIDAD ESCÉNICA: PLANTEAMIENTO DEL MODELO}

La investigación planteada tiene como fin profundizar en el conocimiento de las variables que explican la evolución en el continuum transaccional / relacional a lo largo de la escalera de la fidelidad del cliente que plantean Peck, et al. (1999), Grönroos (1995) y Garbarino y Johnson (1999), en la que, con distintas aproximaciones se supone una relación entre cuatro variables: satisfacción, confianza, compromiso y futuras intenciones de consumo. Las teorías de marketing relacional establecen que los clientes con alto nivel de vinculación no sólo presentan mayores valores en lo que respecta al compromiso y la confianza, sino que éstas pasan a ocupar un lugar central en su comportamiento con respecto a la entidad (Morgan y Hunt, 1994; Sheth y Parvatiyar, 2000; Garbarino y Johnson, 1999).

Las hipótesis de partida de nuestro trabajo pretenden dar un paso más en el análisis de las relaciones organización - cliente para conocer el papel que desempeñan tres constructos clave en la gestión de relaciones con el cliente en el caso de los servicios culturales, éstos son: satisfacción, compromiso y confianza, y en qué medida éstos inciden en el comportamiento de los consumidores de servicios (futuras intenciones de realizar una compra de repetición).

\section{VARIABLES EN EL ESTUDIO}

\subsection{Satisfacción global}

La satisfacción del cliente ha sido tradicionalmente considerada como una de las variables clave en la formación de la fidelidad del cliente. Para Anderson et al. (1994, p. 54) la satisfacción global es una evaluación global basada en la experiencia, adquisición y consumo de un bien o servicio a lo largo del tiempo. Este concepto de evaluación ha de diferenciarse de la evaluación inmediata posterior a la compra (Olivier, 1980, 1993) que recogería el concepto de satisfacción post - compra, que hace referencia a la reacción emocional que el cliente experimenta después de la compra como resultado de la disconformidad (positiva o negativa) entre las expectativas y los sentimientos derivados de su experiencia en una transacción concreta. Esta reacción emocional post-compra y su relación con el consumo va a mostrarse distinta en función del perfil del consumidor. Westbrook y Oliver (1991) identifican modelos diferentes de respuesta ante el consumo, lo que nos indica que existen factores cualitativos de tipo afectivo que coexisten y están relacionados con el continuum unidimensional tradicional desde el que tradicionalmente se ha medido la satisfacción.

De la revisión de la literatura llevada a cabo se desprende que la mayor parte de las investigaciones desarrolladas sobre la satisfacción se basan en el paradigma conformidad / disconformidad, que considera la satisfacción como el resultado de un proceso de comparación entre las expectativas y la experiencia de consumo (Zeithaml et al., 1993). El cliente experimentará una situación de conformidad cuando el servicio recibido iguala las 
expectativas, una situación de disconformidad positiva en el caso de que se excedan sus expectativas y disconformidad negativa cuando el servicio resulte peor de lo esperado.

La aportación de Cronin y Taylor (1992, p. 65) constituye un enfrentamiento a la teoría de Zeithaml, Parasuramen y Berry (1994) al considerar la satisfacción global del cliente (y no la calidad) como la variable de mayor valor para la predicción de las futuras intenciones de consumo de los clientes: "la calidad del servicio es un antecedente de la satisfacción y la satisfacción del servicio del cliente ejerce una mayor influencia en las intenciones de compra que la calidad del servicio". Partiendo de esta perspectiva, los ítems utilizados en el presente trabajo de investigación giran en torno a la consideración y medición de la satisfacción como satisfacción global y satisfacción global comparada, utilizados en otros trabajos desarrollados en el sector de las artes escénicas (Garbarino y Johnson, 1999) y en la línea propuesta por Anderson et al.(1994).

\subsection{Confianza}

Este constructo constituye un valor de referencia clásico en el estudio de las relaciones con clientes (Crosby et al., 1990; Morgan y Hunt, 1994; Berry, 1995; Dyer et al., 1987, entre otros). Moorman et al., 1993, p. 82) definen la confianza como "un deseo de realizar intercambios con un agente del mercado en quien se tiene seguridad". De la misma manera, Morgan y Hunt (1994, p. 23) definen la confianza como "la percepción de credibilidad en la integridad del agente de mercado". Ambas definiciones dejan ver la importancia de este valor, considerado como la base en la construcción de relaciones con el cliente (Berry, 1995; Blois, 1999). Tanto es así, que las teorías de marketing relacional coinciden en considerar que "la existencia de una relación implica algún grado de confianza" (Welch, 2006, p. 140). Desde esta perspectiva, considera la confianza como un continuum que va desde el máximo nivel de confianza hasta el máximo nivel de desconfianza, perspectiva similar a la adoptada por Swift (2001, p. 22), que define la desconfianza como "la asunción de que el agente (de intercambio) perseguirá su propio interés con astucia”, y define la falta de desconfianza como "la confianza en la predictibilidad en el comportamiento del agente (de intercambio)". Es esta la perspectiva que más se acerca a nuestra conceptualización de la confianza. Sin embargo, el concepto de confianza no está exento de cierta controversia en la literatura de marketing sobre los aspectos concretos que debe incluir (Rosenbaum et al., 2006). Leisen y Hyman's (2004) desarrollan una escala original de diez dimensiones para la medida de la confianza en servicios profesionales en la que consideran que la confianza es análoga a los elementos que comprenden la calidad percibida del servicio (la escala SERVQUAL desarrollada por Zeithaml et al., 1996). En esta misma línea, Day y Barksdale (1992) afirman que, para los servicios profesionales, el compromiso resulta muy importante en las fases iniciales de contacto del cliente (fase de selección) y después dejaba de tener importancia en la evaluación del servicio.

Podemos afirmar, por tanto que, en la compleja construcción del concepto "confianza", tienen cabida aspectos relacionados con la calidad percibida del servicio. Incluso algunos autores llegan a afirmar que, para los servicios, sería recomendable incorporar este concepto entre los ítems que miden la calidad percibida del servicio (Leiden y Hyman's 2004: Garbarino y Jonson, 1999). 


\subsection{Compromiso}

De manera similar a la confianza, el compromiso es considerado un constructo fundamental en el desarrollo de relaciones a largo plazo con clientes (Morgan y Hunt, 1994; Dwuer et al., 1987; Russell, 2007). Moorman et al. (1992, p. 316) lo definen como "un deseo duradero de mantener una valorada relación". En este sentido, Russell (2007) considera que los consumidores comprometidos o consumidores "socios" (aquéllos que presentan una relación más cercana con la organización y un mayor nivel de compromiso) se encuentran motivados para mantener la relación debido a la existencia de un sentimiento de vinculación y sinceridad en sus actitudes personales hacia la organización, y desde esta perspectiva afirma que el compromiso resulta vital para la creación y preservación de relaciones con el mercado, representando una faceta actitudinal clave. Fullerton (2005) identifica dos dimensiones bien diferenciadas en la configuración de este constructo: una actitudinal y otra contractual. Esta teoría de la doble dimensión del compromiso ha sido adoptada previamente por otros autores (Bansal et al., Gilliland and Bello, 2002; Gruen et al., 2000; Harrison-Walker, 2001).

En el presente trabajo de investigación hemos adoptado el concepto de compromiso en su perspectiva afectiva, adoptadoa por Morgan y Hunt (1994) quienes emularon la dimensión afectiva del compromiso, adaptando la medida de Allen y Meyer (1990). De esta manera, entendemos que el compromiso afectivo existe cuando el consumidor individual se identifica y se siente vinculado a la organización, a la que considera su "socio" (Fullerton, 2003; Gruen et al, 2000). De esta manera, se entiende que los consumidores se sienten afectivamente comprometidos con el proveedor de servicios cuando les gusta, independientemente del tipo de servicios que se consuman, es decir, independientemente de las experiencias puntuales de una transacción (Fullerton, 2005).

\subsection{Futuras intenciones}

Las intenciones de comportamiento han sido consideradas como variable dependiente en múltiples estudios de investigación en el sector servicios (Boulding et al., 1993; Zeitha$\mathrm{ml}$ et al, 1996), sin embargo, son muy pocos los autores que han aportado información en lo que respecta a su conceptualización y a su medición. Vamos, por tanto a tratar de organizar la información existente al respecto para, en un segundo paso, exponer la perspectiva adoptada en nuestra investigación. Söderlund y Öhman (2005) agrupan las aportaciones realizadas en la conceptualización de las intenciones en dos grandes grupos:

Las intenciones como "planes de futuro".

Ajzen (1991, p. 181) sería un buen exponente de esta perspectiva, que entiende las intenciones como "indicadores de la intensidad con la que una persona está deseando intentar o cuánto esfuerzo está dispuesto a realizar con el fin de hacer algo". Davis y Warshaw (2001, p. 392) comparten esta misma perspectiva de concepto, y entienden que "las intenciones son el grado en el que una persona ha formulado planes conscientes para comportarse de una determinada manera". En esta misma concepción se podría catalogar la propuesta de Malle y Knobe (1997), que consideran que las intenciones incluyen la decisión de realizar un acto. En un estudio empírico los ítems a medir serían del tipo “¿tiene planificado...?”, 
“Tengo la intención de ..." o "Voy a elegir...". Desde esta perspectiva, se puede considera las intenciones como planes de fututo (IPF).

Las intenciones en términos de otras propuestas de futuro.

En este caso nos encontramos ante una situación en la que el individuo se proyecta en la realización de una actividad futura, que pueden ser o bien expectativas o bien deseos. Las expectativas hacen referencia a "la probabilidad que otorga un individuo a la posibilidad de actuar de una determinada manera" (Davis y Warshaw, 2001). En un estudio empírico, los ítems a introducir serían del tipo: "La probabilidad de que haga A es de....". Esta es probablemente la razón por la que las expectativas de comportamiento son denominadas a veces como auto-predicciones (Courneya y McAuley, 1993; Fishbein y Stasson, 1990).

Por último, encontramos el concepto más inhabitual en el ámbito de la investigación de marketing: las intenciones como deseos (ID). Esta perspectiva, utilizada por Fishbein y Stasson (1990) y Smith (1995), considera la intención como un deseo de futuro, que se mediría con un item del tipo "Yo deseo...".

En nuestro trabajo de investigación, hemos adoptado la perspectiva más utilizada en el estudio empírico de las futuras intenciones de los consumidores de servicios: las intenciones como planes de futuro, por considerar que ha sido la más contrastada empíricamente en el ámbito de la investigación científica, habiendo sido mostrada su validez en la medición de los resultados de las organizaciones (Davis y Warshaw, 2001).

\section{DISEÑO DE UN MODELO DE GESTIÓN DE RELACIONES EN LOS SERVI- CIOS: EL COMPROMISO COMO VARIABLE MEDIADORA EN EL COM- PORTAMIENTO FUTURO DE LOS CLIENTES}

La literatura de marketing relacional ha tratado en profundidad las dimensiones que subyacen a los constructos satisfacción, compromiso y confianza, con el fin de obtener información para mejorar las políticas de captación y fidelización de clientes. Las aportaciones realizadas al ámbito de la segmentación de clientes desde el marketing de relaciones apoyan la necesidad de conocer cuáles son las claves en la construcción de una relación con el cliente, y es en este terreno es en el que se desarrolla nuestra investigación, con el fin de aportar información sobre el proceso de construcción de relaciones con el cliente: qué variables influyen y en qué sentido. Asimismo, analizaremos en qué medida estas variables tienen una influencia sobre las intenciones futuras de comportamiento en los consumidores de servicios. A continuación vamos a ir comentando las hipótesis que sustentan el modelo, comenzando por las relaciones entre el constructo satisfacción con el compromiso y la confianza en la organización de servicios.

$H_{1}$. La satisfacción tiene una relación positiva con la confianza.

$\mathrm{H}_{2}$. La satisfacción tiene una relación positiva con el compromiso.

$H_{3}$. La satisfacción no influye de forma directa en las futuras intenciones de consumo.

Diversos estudios analizan esta relación en el entorno de los servicios, concluyendo que la relación entre las variables se mostraba distinta en función del tipo de vínculo desarrollado entre la organización y los clientes. Garbarino y Johnson (1999), en un estudio desarrollado en el sector de las artes escénicas para un teatro de Nueva York, llegan a la 
conclusión de que la satisfacción juega un papel más importante en la predicción de futuras intenciones en los casos en que no existe un vínculo desarrollado con el cliente, mientras que en los clientes que mantenían algún tipo de relación contractual, el compromiso y la confianza actúan como variables mediadoras entre la satisfacción y las futuras intenciones de asistencia.

Oliver (1999) analiza la relación existente entre la satisfacción y la fidelización de clientes. Desde esta perspectiva, la satisfacción constituye un factor importante en la construcción de la lealtad del cliente hacia la organización, pero deja de ser importante en el momento en que éste mantiene una relación más fuerte con la organización (es decir, cuando su compromiso es mayor).

De esta manera, entendemos que está ampliamente contrastado en la literatura de marketing relacional que el primer paso en la construcción de una relación a largo plazo con clientes es necesario un alto nivel de satisfacción, pero una vez iniciada la relación, en determinados tipos de servicios, la satisfacción deja de ser tan importante a favor del compromiso, que adquiere una posición más relevante como variable capaz de predecir los comportamientos futuros del cliente.

El sector de servicios culturales, en el que se desarrolla nuestra investigación empírica, se caracteriza por contar con clientes de consumo intenso y altamente comprometidos con la organización (Quero, 2003), lo que nos indica que la satisfacción no es un indicador suficiente para predecir las intenciones futuras de consumo, es necesario un vínculo mayor como el compromiso. Además, las peculiaridades del producto hacen que, en muchas ocasiones, en el grupo de consumidores menos frecuentes, el motivo de consumo del servicio cultural resida en el proveedor (una determinada compañía, un artista conocido, etc.), de tal manera que los clientes no realizarán un consumo de repetición si no se vuelve a realizar la oferte del mismo proveedor.

\section{$H_{4}$. La confianza mantiene una relación positiva con el compromiso.}

$H_{5}$. La confianza no influye en la predicción de las futuras intenciones de asistencia.

Las relaciones entre confianza y compromiso han sido tratadas en profundidad en diversos sectores económicos desde que empieza a desarrollarse el marketing relacional. Morgan y Hunt (1994) fueron los pioneros en desarrollar las dimensiones e implicaciones de esta relación, así como los beneficios que de ella se derivaban en las relaciones entre fabricantes y distribuidores. Desde esta aportación, muchos autores han confirmado en distintos ámbitos su existencia (Rusell, 2007; MacMillan et al, 2005).

La relación entre el constructo confianza y las futuras intenciones de consumo no ha sido tan ampliamente tratada en la literatura de marketing de forma exclusiva, pero sí como parte de sistemas más amplios de relaciones, como los desarrollados por Woodside, et al. (1989). En el ámbito de los servicios de artes escénicas Garbarino y Johnson (1999) llegan a la conclusión de que, sólo para el caso de los consumidores que mantienen un vínculo con la organización (abono o similar), esta relación se muestra positiva. Para los consumidores esporádicos u ocasionales, esta relación no es significativa.

El estudio realizado por Rosenbaum et al. (2006) para el sector de los servicios profesionales pone de manifiesto asimismo la incapacidad de la confianza para predecir las futuras intenciones de los consumidores, alegando que los consumidores probablemente dan por garantizada la confianza si ya han desarrollado un vínculo de compromiso con 
ésta, y por lo tanto un elevado nivel de confianza no tiene por qué ser capaz de asegurar un consumo futuro.

$H_{6}$. El compromiso influye de forma directa y positiva sobre las intenciones futuras de consumo.

El compromiso se perfila como la variable con mayor capacidad de influir sobre las futuras intenciones de asistencia. Entendiendo el compromiso desde la perspectiva afectiva, se considera que la identificación del consumidor con la organización, con sus valores, y su implicación en lo que afecta a la organización de servicios es la única variable que mantiene una relación positiva con la asistencia futura de la audiencia. Fullerton (2005) analiza en profundidad esta relación para tres sectores de servicios (financieros, telecomunicaciones y alimentación) y concluye que los consumidores que sienten una "conexión psicológica" con su proveedor de servicios, actuarán como prescriptores para la organización. En esta misma línea que relaciona el compromiso afectivo y los comportamientos futuros en la industria de servicios se desarrollan las aportaciones de Rusell, 2007; Gruen et al., 2000 y Harrison-Walker, 2001.

Garbarino y Jonson (1999) demuestran que, para el sector de los servicios de exhibición de artes escénicas, los consumidores que registran un fuerte vínculo relacional, el compromiso constituye una excelente variable de predicción de las intenciones futuras de consumo. De manera similar, Rosenbaum et al. (2006) contrastan la capacidad del compromiso para predecir el comportamiento futuro de repetición de compra.

Figura 1

Modelo de gestión de las relaciones en los servicios

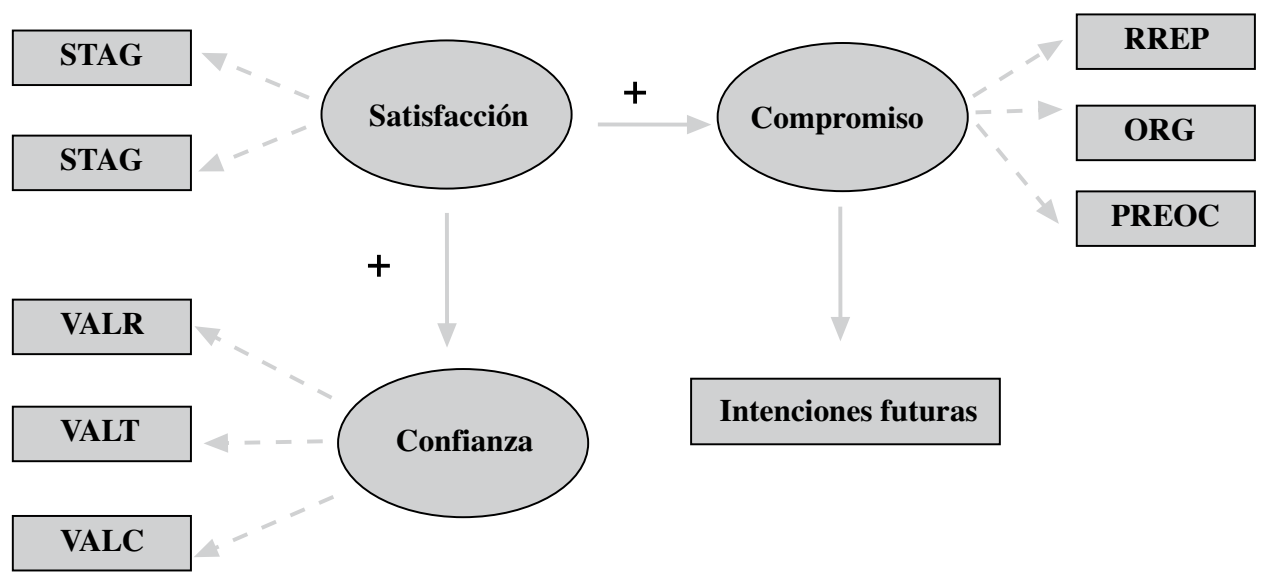

\section{METODOLOGÍA Y MEDIDA DE LAS VARIABLES}

Para la constrastación de las hipótesis se realizó una encuesta personal cerrada, en la entrada o salida de los teatros. En la realización participaron 150 teatros de toda España y 
1005 consumidores. En una primera revisión de los datos, resultó especialmente destacado el escaso porcentaje de consumidores con vínculo formal con la organización (abonados o similares), que representaban tan sólo el 7,7 \% de la población, por lo que decidimos centrarnos en los consumidores no abonados, que representan el 92,3\% (927 encuestados) y que resultaban más representativos del comportamiento de la población. El motivo de seleccionar los consumidores de forma aleatoria en el lugar de consumo, se debe a que el presente trabajo de investigación trata de arrojar luz sobre relaciones con la organización, y una encuesta al público general podría introducir un alto grupo de no consumidores que no nos aportaría información útil al estudio. La selección de los 150 teatros fue realizada de forma directa por parte de los investigadores, para asegurar la presencia de todos los principales teatros de todas las ciudades de España. La distribución de consumidores en la muestra fue realizada estableciendo cuotas por edad, sexo y geográfica correspondientes a la media nacional. El trabajo fue llevado a cabo por TNS España entre los meses de noviembre y febrero, por considerar que son meses en los que se intensifica el consumo de artes escénicas en las ciudades, en opinión de los directores de los teatros participantes.

La medición de la satisfacción global se realizó con dos ítems previamente utilizados por Garbarino y Johnson (1999) para evaluar la misma categoría de servicio en EEUU. Los ítems que integran el compromiso afectivo fueron seleccionados de los estudios realizados por Fullerton (2005), Allen y Meyer (1990) y Garbarino y Johnson (1999). La medición de la confianza se realiza con tres ítems adaptados de Morgan y Hunt (1994), y Garbarino y Johnson (1999). En lo que respecta a las futuras intenciones de los consumidores, consideramos apropiada la inserción de una variable dummy, que obligara al encuestado a decantarse por una alternativa específica sin generar dudas sobre si se planteaba el concepto de futuras intenciones como expectativas o deseos. El motivo de esta elección lo encontramos en el concepto adoptado de futuras intenciones (IP, Intenciones como planes), que considerábamos el más apropiado para el contenido de nuestro trabajo. La opción de una variable dummy se debe a que, tal y como proponen Davis y Warshaw (2001 p. 404) "Si las intenciones de comportamiento futuro se miden en escalas superiores a 3 puntos, los encuestados probablemente pensarán que se les está solicitando información sobre sus expectativas de comportamiento. De hecho, la intención de hacer o no hacer algo es difícil incluso de medir en una escala de 3 puntos". Los ítems de la investigación quedan recogidos en la tabla 1 .

Tabla 1

Escalas de medida de los constructos

\begin{tabular}{|c|l|c|}
\hline Constructo & \multicolumn{1}{|c|}{ Items } & $\begin{array}{c}\text { Denominación } \\
\text { abreviada }\end{array}$ \\
\hline \multirow{3}{*}{ Satisfacción } & $\begin{array}{l}\text { Valore, en una escala de 1 a 7, su nivel de satisfacción por lo } \\
\text { que respecta a esta entidad. }\end{array}$ & SATG \\
\cline { 2 - 3 } & $\begin{array}{l}\text { Valore, en una escala de 1 a 7, su nivel de satisfacción por lo } \\
\text { que respecta a esta entidad con respecto a otras entidades de } \\
\text { ocio existentes en la misma ciudad. }\end{array}$ & SATC \\
\hline
\end{tabular}




\begin{tabular}{|c|c|c|}
\hline Constructo & Items & $\begin{array}{c}\text { Denominación } \\
\text { abreviada }\end{array}$ \\
\hline \multirow{3}{*}{ Confianza } & $\begin{array}{l}\text { Valore, en una escala de } 1 \text { a } 7 \text { el nivel de influencia que tienen las } \\
\text { recomendaciones emitidas por la propia entidad o personal de la } \\
\text { entidad en su decisión de asistencia a una representación escénica. }\end{array}$ & VALR \\
\hline & $\begin{array}{l}\text { Valore, en una escala de } 1 \text { a } 7 \text { su nivel de acuerdo con las } \\
\text { siguiente frase: "Esta organización desarrolla bien su trabajo } \\
\text { como entidad cultural". }\end{array}$ & VALT \\
\hline & $\begin{array}{l}\text { Valore, en una escala de } 1 \text { a } 7 \text { su nivel de acuerdo con las } \\
\text { siguiente frase: "En general, la calidad de los espectáculos } \\
\text { emitidos por esta entidad es elevada". }\end{array}$ & VALC \\
\hline \multirow{3}{*}{ Compromiso } & $\begin{array}{l}\text { ¿Qué tipo de comentarios suele realizar en su entorno sobre } \\
\text { esta entidad? } \\
\text { Muy negativos / negativos / ninguno en especial / positivos / } \\
\text { muy positivos. }\end{array}$ & REFP \\
\hline & $\begin{array}{l}\text { Valore, en una escala de } 1 \text { a } 7 \text { su nivel de acuerdo con las } \\
\text { siguiente frase: "Me siento orgulloso de ser consumidor / abo- } \\
\text { nado / socio de esta entidad". }\end{array}$ & ORG \\
\hline & $\begin{array}{l}\text { Valore, en una escala de } 1 \text { a } 7 \text { su nivel de acuerdo con las } \\
\text { siguiente frase: Me preocupa lo que pueda afectar a esta entidad } \\
\text { a largo plazo". }\end{array}$ & PREOC \\
\hline $\begin{array}{l}\text { Futuras } \\
\text { intenciones }\end{array}$ & $\begin{array}{l}\text { ¿Tiene planificado asistir a otras representaciones en la presente } \\
\text { temporada? }\end{array}$ & FI \\
\hline
\end{tabular}

\section{ANÁLISIS Y RESULTADOS}

Para el análisis de datos se realizó un análisis de ecuaciones estructurales haciendo uso del programa informático AMOS 16.0.

Los índices de ajuste global del modelo arrojan valores que le otorgan un elevado valor de significación: el estadístico Chi-Cuadrado $\left(\mathrm{X}^{2}\right)$ registra un valor de 61,118 con 22 grados de libertad y el nivel de significación no es $\leq 0,01$. Este indicador mide la distancia ente la matriz de datos inicial y la estimada por el modelo. Un importante inconveniente de este estadístico es que para muestra suficientemente amplias, como es nuestro caso $(n=927)$, se incrementa la probabilidad de rechazar el modelo aunque la diferencia entre las matrices sean mínimas. Por este motivo, recurrimos a otros estadísticos de medida que nos indiquen la validez del modelo.

El residuo cuadrático medio (RMSR) es la raiz cuadarada de la medida de los residuos al cuadrado (una media de los residuos entre las matrices de entrada estimadas y observadas). Esta medida de ajuste fue introducida por Steiger (1990) para intentar eliminar el inconveniente que presentaba la $\mathrm{X}^{2}$ cuando la muestra era lo suficientemente grande. Valores de RMSEA inferiores a 0,05 e incluso a 0,08 son indicativos de un buen ajuste del modelo en la población (Luque, 2000; Browne y Cudeck, 1993). En el modelo desarrollado en nuestra investigación arroja un valor de 0,44 lo que nos indica un buen ajuste global. El índice de juste normal o Delta 1 (NFI) es una comparación relativa del modelo propuesto al modelo nulo (Bentler y Bonett, 1980); el rango de valoración de este índice 
está entre 0 y 1 , siendo recomendable valores superiores a 0,90 (en el caso de nuestro modelo NFI=0,971, superando con creces el umbral que determina la validez del modelo. El índice de ajuste relativo (RFI o Rho) premia a los modelos más parsimoniosos o simples, si bien también depende del tamaño muestral y proporciona valores próximos a la unidad a medida que le modelo va alcanzando un buen ajuste (en el modelo propuesto RFI=0,941). El Índice de ajuste incremental (IFI o Delta 2) propuesto por Bollen (1989) reduce la dependencia de NFI respecto al tamaño muestral, por lo que resulta especialmente apropiado para nuestra investigación. Para nuestro modelo $\mathrm{IFI}=0,981$, dato que se sitúa muy cercano a la unidad, lo que nos indica un buen ajuste del modelo. En lo que respecta a las medidas de parsimonia, que relacionan la calidad del ajuste del modelo al número de coeficientes estimados exigidos para conseguir este nivel de ajuste, observamos los siguientes valores de indicadores, que superan sobradamente el límite que marca la aceptación del modelo: PNFI=0,475; PRATIO=0,789; $\mathrm{AIC}=125,118$; Por último, CN Hoetler sugiere el tamaño que una muestra debe alcanzar en orden al aceptar el ajuste de un modelo dado sobre una base estadística. Para nuestro caso, y en la restricción más estricta $(0,01)$, el tamaño de la muestra requerido es de 611 , valor que supera sobradamente la investigación realizada. La tabla 2 recoge un resumen del total de indicadores del modelo comentados.

Tabla 2

Indicadores de medición del ajuste del modelo

\begin{tabular}{lcl}
\hline \multicolumn{1}{c}{ Indicadores de bondad del ajuste } & Valores & \multicolumn{1}{c}{ Niveles de aceptación } \\
\hline Chi-cuadrado & 61,118 & Depende de tamaño muestral \\
NCP & 39,118 & Depende del modelo \\
RMSEA & 0,044 & $\leq 0,05$ \\
ECVI & 0,135 & $\sim 0$ \\
$\quad$ & & \\
$\quad$ Medidas incrementales del ajuste & & \\
NFI & 0,971 & $\geq 0,9$ \\
RFI & 0,941 & Valores próximos a 1 \\
CFI & 0,981 & Valores próximos a 1 \\
IFI & 0,981 & Valores próximos a 1 \\
TLI & 0,962 & $\geq 0,9$ \\
$\quad$ & & \\
Medidas de ajuste de parsimonia & & En comparación de modelos alternativos, arro- \\
PNFI & 0,475 & jaba el valor más elevado. \\
AIC & & Valor alto en comparación al modelos alterna- \\
Critical N & 125,118 & tivos. \\
\hline
\end{tabular}

El lo que respecta a los ítems recogidos en las escalas de medida, se considera aceptable el nivel de validez convergente, ya que todos los estimadores de los factores son altamente 
significativos y presentan valores superiores a 0,6 para los estimadores de las correlaciones múltiples al cuadrado para todas las variables (Bagozzi y Yi, 1988). La validez discriminante fue evaluada atendiendo a los métodos diseñados por Fornell y Larker (1981), dando como resultado un alto nivel de validez discriminante basada en el análisis de la correlación al cuadrado entre pares de constructos, que resultó ser inferior a la varianza extraída media de cada constructo.

El análisis de las hipótesis del modelo arrojan asimismo valores de relación significativos para las tres hipótesis representadas. $\mathrm{La} \mathrm{H}_{1}$, que mide la relación positiva entre satisfacción y confianza resulta significativa $(p<0,000, \beta=0,468, C R=7,122)$. Los valores de $C R$ superiores a 1,96 son considerados como significativos (Anderson y Gerbing, 1991). La $\mathrm{H}_{2}$, que establece una relación directa y positiva entre satisfacción y compromiso, también se confirma $(p<0,000, \beta=0,437, \mathrm{CR}=4,217)$. $\mathrm{La} \mathrm{H}_{3}$, que niega la relación entre satisfacción y futuras intenciones, también se confirma. El bajo valor de CR $(0,491)$ es considerablemente inferior al umbral de 1,96, y el nivel de significación supera el umbral de $p \geq 0,05$ con un valor $\mathrm{p}=0,491$.

Desde el constructo confianza también se confirman las dos hipótesis planteadas: en primer lugar la $\mathrm{H}_{4}$, que relaciona positivamente confianza y compromiso $(p<0,000$, $\beta=0,722, C R=3,603$ ) y la $\mathrm{H}_{5}$, que establece que no hay relación entre la confianza y las futuras intenciones de asistencia ( $\mathrm{p}=0,90 \geq 0,05, \beta=-0,277, \mathrm{CR}=-1,697)$.

La última hipótesis planteada en el modelo $\left(\mathrm{H}_{6}\right)$ establece una relación positiva y directa entre el compromiso y las futuras intenciones de asistencia. Los estadísticos nos permitiron eceptar la hipótesis para un nivel de confianza del 95,5\% $(\mathrm{p}=0,021<0,005, \beta=0,360$, $\mathrm{CR}=2,303$ ). La tabla 3 recoge los valores de los estimadores de las hipótesis.

Tabla 3

Estimadores de las hipótesis

\begin{tabular}{lcccc}
\hline \multicolumn{1}{c}{ Relaciones de las hipótesis } & Estimadores & $\mathrm{SE}$ & $\mathrm{CR}$ & $p$ \\
\hline $\mathrm{H}_{1}:$ Satisfacción $\rightarrow$ Confianza & 0,468 & 0,066 & 7,122 & $<0,000$ \\
$\mathrm{H}_{2}:$ Satisfacción $\rightarrow$ Compromiso & 0,437 & 0,104 & 4,217 & $<0,000$ \\
$\mathrm{H}_{3}:$ Satisfacción $\rightarrow$ Futuras intenciones & $-0,063$ & 0,092 & 0,688 & 0,491 \\
$\mathrm{H}_{4}:$ Confianza $\rightarrow$ Compromiso & 0,722 & 0,200 & 3,603 & $<0,000$ \\
$\mathrm{H}_{5}:$ Confianza $\rightarrow$ Futuras intenciones & $-0,277$ & 0,163 & $-1,697$ & 0,90 \\
$\mathrm{H}_{6}:$ Compromiso $\rightarrow$ Futuras intenciones & 0,360 & 0,157 & 2,303 & $0,021<0,005$ \\
\hline
\end{tabular}

Las tablas 4 y 5 recogen los valores de los estimadores de las variables observables que integran los constructos y los indicadores estadísticos que indican el nivel de significación para cada uno de los indicadores. Los altos valores de CR (t) y el nivel de significación, que resulta ser inferior a 0,01 para todas las variables nos permiten confirmar su alto valor de representación en el modelo. 
Tabla 4

Coeficientes de regresión estandarizados

\begin{tabular}{lc}
\hline \multicolumn{1}{c}{ Relaciones entre variables observadas y constructos } & Estimador \\
\hline Satisfacción $\rightarrow$ Compromiso & 0,499 \\
Satisfacción $\rightarrow$ SATC & 0,755 \\
Satisfacción $\rightarrow$ SATG & 0,845 \\
Confianza $\rightarrow$ VALC & 0,733 \\
Confianza $\rightarrow$ VALT & 0,829 \\
Confianza $\rightarrow$ VALR & 0,274 \\
Compromiso $\rightarrow$ REFP & 0,595 \\
Compromiso $\rightarrow$ ORG & 0,677 \\
Compromiso $\rightarrow$ PROC & 0,460 \\
\hline
\end{tabular}

Tabla 5

Indicadores de los componentes de los constructos

\begin{tabular}{|c|c|c|c|c|}
\hline Variable observada & Estimador & SE & $\mathrm{CR}$ & $p$ \\
\hline SATC & 5,083 & 0,042 & 121,321 & $<0,000$ \\
\hline SATG & 5,339 & 0,039 & 138,631 & $<0,000$ \\
\hline REFP & 3,751 & 0,025 & 148,213 & $<0,000$ \\
\hline ORG & 4,697 & 0,057 & 82,522 & $<0,000$ \\
\hline PREOC & 5,070 & 0,059 & 86,140 & $<0,000$ \\
\hline VALC & 5,361 & 0,039 & 136,014 & $<0,000$ \\
\hline VALT & 5,343 & 0,041 & 129,644 & $<0,000$ \\
\hline VALR & 3,636 & 0,061 & 59,150 & $<0,000$ \\
\hline Futuras intenciones & 0,745 & 0,015 & 48,249 & $<0,000$ \\
\hline
\end{tabular}

\section{VALORACIÓN DE LOS RESULTADOS Y CONCLUSIONES}

El modelo de gestión de las relaciones propuesto profundiza en el conocimiento de las relaciones con clientes en el sector servicios, aportando información sobre cómo se construyen las relaciones entre tres variables clave en la gestión de relaciones con clientes: satisfacción, compromiso y confianza. Aunque muchos estudios han analizado la relación entre estas variables y la percepción de calidad del servicio, la vinculación con las futuras intenciones de consumo que realizamos en este estudio no ha sido tratada previamente. De esta manera, adoptando el concepto de futuras intenciones como "planes de futuro del cliente" (Davis y Warshaw, 2001; Söderlund, M. y Öhman, 2005), llegamos a la conclusión 
de que la única variable capaz de incidir sobre el comportamiento futuro del cliente es el compromiso, tal y como nos indica la confirmación de la hipótesis $\mathrm{H}_{6}$.

La hipótesis $\mathrm{H}_{3}$ confirma la inexistencia de relación entre satisfacción y futuras intenciones. Este resultado nos indica que, tal y como propone Oliver (1999), la satisfacción resulta muy importante en las primeras fases de construcción de una relación con el cliente, pero una vez superada esa fase inicial, la construcción de una buena relación con la organización de servicios es la que puede asegurar, no sólo que el cliente vuelva (futuras intenciones), también generará las múltiples ventajas que se derivan de la fidelización de clientes: difusión de referencias positivas, insensibilización ante acciones de la competencia e implicación en el futuro de la entidad, etc. Por lo tanto, la satisfacción no es que deje de ser importante, sino que el cliente que mantiene una relación fuerte con la organización da por hecho la existencia de un nivel de satisfacción adecuado a su demanda e incluso, si se produce algún tipo de fallo en la entrega del servicio, el cliente será mucho más benevolente en la evaluación de la calidad del servicio, ya que si el nivel de compromiso que éste siente por la organización es elevado, al cliente le importará lo que pueda pasar con la entidad a largo plazo.

$\mathrm{La}_{5}$ confirma la no relación entre confianza y futuras intenciones. En teste caso tampoco debemos suponer que la confianza no sea importante en la construcción de una relación entre cliente y proveedor de servicios, pero en determinados servicios como los que nos ocupan es posible que ésta se de por supuesta, no asegurando por sí sola que el cliente vaya a repetir el consumo en un futuro próximo.

\subsection{Implicaciones empresariales más destacadas del estudio}

Estos datos resultan de interés en el diseño de estrategias de marketing de las empresas de servicios, ya que si lo que la empresa pretende es gestionar las relaciones con el cliente con el fin de ir incrementando los vínculos de éste con la organización, siguiendo la estrategia de la "Escalera de fidelidad del marketing relacional" propuesta por Christopher et al. (2007), hace falta algo más que un cliente satisfecho, es necesario vincular emocionalmente al cliente con la organización, implicarlo más directamente en sus acciones y en sus valores. Tan sólo este tipo de clientes socios o amigos de la empresa, que se sienten emocionalmente vinculados a ella nos asegurarán un consumo futuro.

El modelo también nos descubre cómo, la satisfacción y la confianza son importantes en el proceso de construcción del compromiso en los servicios. Las hipótesis $\mathrm{H}_{2} \mathrm{y}_{4} \mathrm{H}_{4}$ demuestran que existe una relación directa y positiva entre estas variables, lo que nos indica que en el complejo proceso de construcción del compromiso es importante contar con un alto nivel de satisfacción y de confianza. En la literatura de marketing relacional también abunda información sobre la importancia de la satisfacción y la confianza en la construcción de relaciones a largo plazo (Zeithaml et al., 1996), pero el trabajo de investigación desarrollado demuestra que no es suficiente, resulta necesario, además, vincular al cliente con la organización. Esa vinculación va más allá de formalismos del tipo contractual, como un abono, necesita una vinculación del tipo emocional, que involucre en mayor medida al cliente con la organización. 
Del análisis de los datos de la investigación empírica podemos concluir que, en la medida en que una organización de servicios pretenda vincular a los clientes y asegurarse una intención positiva de consumo futuro, es importante que, con los clientes que no presentan una vinculación formal con la organización (tipo abonos o similares) que le asegure un nivel de consumo futuro, se desarrollen acciones dirigidas a alimentar la identificación del cliente con la organización, de tal manera que éste se vea afectado por lo que suceda a la organización y recomiende en su entorno el consumo del servicio.

La satisfacción y la confianza en el servicio es importante como instrumentos para la construcción del compromiso con los clientes. Los resultados de la investigación no restan valor a estas variables, muy por el contrario, resultan una condición sine qua non para iniciar una relación a largo plazo con el cliente.

También sería interesante plantear, a la vista de los resultados, si el compromiso formal (un abono, una tarjeta o cualquier otra forma de asociación tradicional) es la mejor forma de medir o evaluar el compromiso. Es más, dependiendo de las circunstancias, hay estudios que plantean incluso un tipo de vínculo que puede desarrollar relaciones negativas, en las que el cliente se siente "capturado" en la relación (Fulleton, 2005). Por lo tanto, no debemos entender que la vinculación formal a una entidad de servicios termina con la asociación del cliente; tras el término de "socio" se introducen valores mucho más importantes como la difusión de referencias positivas, sentimiento de orgullo e identificación o preocupación por lo que afecte a la organización.

\subsection{Futuras investigaciones}

El modelo planteado constituye una aportación a la medición de los Rendimientos del Marketing Relacional que en tantas ocasiones se han planteado. Si bien nos ha permitido aportar información sobre cómo construir relaciones fructíferas para la organización y para el cliente, sería importante desarrollar en futuras investigaciones los constructos, analizando otras dimensiones planteadas en la teoría sobre cada uno de ellos y, en lo que respecta al estudio empírico, contrastar la validez del modelo en otros servicios distintos del entorno que ofrece la exhibición de artes escénicas en España.

\section{REFERENCIAS BIBLIOGRÁFICAS}

AJZEN, I. (1991): “The theory of planned Behavior", Organizational Behavior and Human Decision Processes, Vol. 50, pp. 179 - 211.

ALLEN, N. Y MEYER, J. (1990): “The measurement and antecedents of affective, continuance and normative commitement to the organization", Journal of Occupational Psychology, Vol. 63, No. 1 , pp. $1-18$.

ANDERSON, E. W., FORNELL, C. Y LEHMANN, D.R. (1994): "Customer Satisfaction, Market Share, and profitability: Findings from Sweeden", Journal of Marketing, Vol. 58, pp. 53 - 66.

ANDERSON, J. C. Y GERBING, D. W. (1988): "Structural Equation Modeling in Practice: A Review and Recommended Two - Step Approach", Psychological Bulletin, No 103 (3), pp. 411 $-423$.

BAGOZZI, R. P. (1975): “Marketing as Exchange”, Journal of Marketing, No 40 (Julio), pp. 17 - 28. 
BAGOZZI, R. Y YI, Y. (1988): "On the evaluation of structural equation models", Journal of the Academy of Marketing Science, Vol. 16, No 1, pp. 74 - 94.

BANDURA, A. (1986): "Social Foundations of Thought and Action: A Social Cognitive Theory", Prentice Hall, New Jersey.

BANSAL, H. Y TAYLOR, S. (1999): “The service switching model (SMM): A model of switching behaviour in services industries", Journal of Service Research, Vol. 2, No 2, pp. 200 - 18.

BENTLER, P. M. Y BONNETT, D. G. (1980): "Significance Tests and Goodness of Fit in the Analysis of Covariance Structures", Psychological Bulletin, No 88, pp. 588 - 606.

BERRY, L. (1995): "Relationship marketing of services: growing interest, emerging perspectives", Journal of the Academy of Marketing Science, Vol. 23, No 4, pp. 236 - 45.

BLOIS, K. (1999): "Trust in business relationships: an evaluation of its status", Journal of Management Studies, Vol. 36, No 2, pp. 197 - 215.

BOLLEN, K. A. (1989): Structural Equation with Latent Variables, Ed. Wiley and Sons, New York.

BONNEMAIZON, A., COVA, B. Y LOUYOT, M.C. (2007): "Relationship Marketing in 2015: A Delphy Approach", European Management Journal, No 1, Vol. 25, pp. 50 - 59.

BOULDING, W., KALRA, A., STAELIN, R. Y ZEITHAML, V. A. (1993): "A Dynamic Process Model of Service Quality: from Expectations to Behavioral Intentions", Journal of Marketing Research, Vol. 30, febrero, pp. 7 - 23.

BROWNE, M. W. Y CUDECK, R. (1989): Single sample cross - validation indices for covariance structures", Multivariate Behavioral Research, No. 24, pp. 445 - 455.

CHRISTOPHER, M., PAYNE, A. Y BALLANTYNE, D. (2007): "Relationship Marketing”, Ed. Butterworth Heinemann, Oxford.

COLBERT, F. (2001): "Marketing Culture and the Arts", Second edition, Presses HEC, Montréal.

COURNEYA, K.S. Y MCAULEY, E. (1993): "Predicting Physical Activity from Intention: Conceptual and Methodological Issues", Journal of Sport and Exercise Psichology, Vol. 15, pp. 50 - 62.

CRONIN, J. J. (JR.) Y TAYLOR, S. A. (1992): "Measuring Service Quality: A Reexamination and Extensión", Journal of Marketing, Vol. 56 (julio), pp. 55 - 68.

CROSBY, L. A.; EVANS, K. R.; COWLES, D. (1990): "Relationship Quality in Services Selling: An Interpersonal Influence Perspective", Journal of Marketing, No. 54 (Julio), pp. 68 - 81.

DAVIS, F. D. Y WARSHAW, P. R. (2001): "What do Intention Scales Measure?", The Journal of General Psychology, 119, No. 4, pp. $391-407$.

DAY, E. Y BARKSDALE, H. C. JR. (1992): "How Firms Select Professional Services”, Industrial Marketing Management, No. 21 (mayo), pp. 85 - 91.

DWYER, F. R.; SCHURR, P.H.; OH, S. (1987): “Developing Buyer - Seller Relationships”, Journal of Marketing, Vol. 51 (abril), pp. $11-27$.

FISHBEIN, M. Y STANSSON, M. (1990): "The role of desires self - predictors, and perceived control in the prediction of training session attendance", Journal of Applied Social Psychology, Vol. 20, No. 3, pp. 173 - 198.

FISHBEIN, M. Y STASSON, M. (1990): “The Role of Desires Self-Predictiona and Perceived Control in the Prediction of Training Session Attendance", Journal of Applied Social Psychology, Vol. 20, No. 3, pp. 173 - 198.

FORNELL, C. Y LARCKER, D. (1981): "Evaluating structural equation models with unobservable variables and measurement error", Journal of Marketing Research, Vol. 18, No. 1, pp. 39 - 50.

FULLERTON, G. (2003): "When does commitment lead to loyalty", Journal of Service Research, No. 4, Vol. 5, pp. 333 - 344.

FULLERTON, G. (2005): "How commitment both enables and undermines marketing relationships", European Journal of Marketing, No. 11/12, Vol. 39, pp. 1372 - 1388.

GARBARINO, E. Y JONSON, M. S. (1999): "The Different Roles of Satisfaction, Trust and Commitment in Customer Relationships”, Journal of Marketing, Vol. 63 (abril), pp. 70 - 87. 
GILLIAND, D. Y BELLO, D. (2002): "The two sides to attitudinal commitment: the effect of calculative and loyalty commitment on enforcement mechanisms in distribution channels", Journal of the Academy of Marketing Science, Vol. 30, No. 1, pp. 24 - 43.

GRÖNROOS, C. (1995): "Relationship marketing: the strategy continuum", Journal of the Academy of Marketing Science, vol. 23, No. 4, pp. $252-256$.

GRUEN, T., SUMMERS, J. Y ACITO, F. (2000): "Relationship Marketing Activities, commitment and membership behaviours in professional associations", Journal of Marketing, Vol. 64, No. 3, pp. $34-49$.

GUMMESSON, E. (2004): Many to Many Marketing. Malmo: Liber.

GUMMESSSON, E. (2008): Total Relationship Marketing, Butterworth-Heinemann, Oxford.

HARRISON - WALKER, J. (2001): "The measurement of word-of-mouth communication and an investigation of service quality and customer commitment as potential antecedents", Journal of Service Research, Vol. 4 No. 1, pp. 60 - 75 .

HENNING-THURAU, T., GWINNER, K.P. Y D.D. GREMLER (2002): “Understanding Relationship Marketing Outcomes: An Integration of Relational Benefits and Relationship Quality”, Journal of Service Research, No. 4 (febrero), pp. $230-248$.

HILL, E.; O’SULLIVAN, C. Y O’SULLIVAN, T. (1995): “Creative Arts Marketing”, Ed. Butterworth - Heinemann, Oxford.

HIRSCHMAN, E.C. (1983): "Aesthetics, Ideologies and the Limits of the Marketing Concept", Journal of Marketing, Vol. 47 (verano), pp. 45 - 55.

HOUSTON, F. S.; GASSEMHEIMER, J. B. (1987): "Marketing and Exchange”, Journal of Marketing, Vol. 51, Octubre, págs. 3 - 18.

HUNT, S. D. (1983): "General Theories and the Fundamental Explanada of Marketing”, Journal of Marketing, Vol. 47, (otoño), pp. 9 - 17.

KOTLER, P. (1967): "Marketing Management Analysis, Planning and Control", Ed. Englewood Cliffs, Prentice Hall, NJ.

LEISEN, B. Y HYMAN, M. R. (2004): "Antecedents and Consequences of Trust in Service Provider: The Case of Primary Care Physicians”, Journal of Business Research, No. 57, septiembre, pp $990-999$.

LOVELOCK, C. Y GUMMESSON, E. (2004): "Whither Services Marketing? In Search of a New Paradigm and Fresh Perspectives", Journal of Service Research, No. 1, Vol. 7, pp. 20 - 41.

LUQUE, T. (2000): “Técnicas de análisis de datos en investigación de mercados”, Ed. Pirámide, Madrid.

MACMILLAN, K., MONEY, K., MONEY, A. Y DOWNING, S. (2005): "Relationship Marketing in the not - for - profit sector: an extension and application of the commitment - trust theory", Journal of Business Research, No. 58, pp. 806 - 818.

MALLE, B. F. Y KNOBE, J. (1997): “The Folk Concept of Internationality”, Journal of Experimental Social Psychology, Vol. 33, pp. $101-121$.

MCCARTHY, E. J. (1960): "Basic Marketing, A Managerial Approach”, Homewood IL: Richard D. Irwin.

MOORMAN, C., ZALTMAN, G. Y DESHPANDE (1992): "Relationships Between Provides and Users of Market Research: The Dynamics of Trust Within and Between Organizations", Journal of Marketing Research, No. 29 (agosto), pp. 314 - 328.

MOORMAN, C.; DESHPANDÉ, R. Y ZALTMAN, G. (1993): "Factors Affecting Trust in Market Relationships", Journal of Marketing, Vol. 57 (enero), pp. 81 - 101.

MORGAN, R.M. Y HUNT S.D. (1994): "The commitment-trust theory of relationship marketing", Journal of Marketing, Vol. 58, pp. 20 - 38.

OLIVER (1999): “Whence Consumer Loyalty?”, Journal of Marketing, Vol. 63, Special Issue, págs. $33-44$. 
OLIVER, R. L. (1977): "A Cognitive Model of the Antecedents and Consequences of Satisfaction Desions", Journal of Marketing Research, 17, November, pp. 460 - 469.

OLIVER, R. L. (1993): "A Conceptual Model of service Quality and Service Satisfaction" en "Advances in Services Marketing and Management", de Swartz, T. A.; Bowen, D. E. y Brown, S. W., Ed. JAI Press, Greenwich, CT, pp. 65 - 86.

QUERO, M.J. (2003): “Marketing Cultural. El enfoque relacional en las entidades escénicas" Ed. Red Española de Teatros, Auditorios y Circuitos de Titularidad Pública, Madrid.

QUERO, M.J. (2007): "Relationship Marketing and Services Marketing: Two Convergent Perspectives for Value Creation in the Cultural Sector". International Review on Public and Non Profit Marketing, Vol. 4, nº 1/2, págs. $101-115$.

QUERO, M. J. Y VENTURA, R. (2009): "The Role of Stakeholders in the Management of Cultural Organisations. The case of performing Arts Organisations in Spain", Journal of Relationship Marketing, Vol. 8, pp. $19-37$.

ROSENBAUM, M. S.; MASSIAH, C. Y JACKSON, D. W. (2006): “An Investigation of Trust, Satisfaction and Commitment on Repurchase Intentions in Professional Services", Services Marketing Quarterly, Vol. 27, No. 39, pp. 115 - 135.

RUSSELL, L. (2007): "Relationship drivers of customer commitment", Journal of Marketing Theory and practice", Vol. 15, No. 4, pp. 315 - 333.

SAN MARTÍN, S., GUTIERREZ, J. Y CAMARERO, M. (2004): "Trust as the Key to Relational Commitment”, Journal of Relationship Marketing, No. 3, Vol. 1, pp. 53 - 73.

SHETH, J. N. Y PARVATIYAR, A. (2000): “Handbook of Relationship Marketing”. Sage Publications, California.

SÖDERLUND, M. (2002): "Customer satisfaction and its effects on different behavioural intention constructs", Journal of Customer Behaviour, Vol. 1, pp. 145 - 166.

SÖDERLUND, M. Y ÖHMAN, N. (2005): “Assesing behavior before it becomes behavior”, International Journal of Service Industry Management, Vol. 16, No. 2, pp. 169 - 185.

STEIGUER, J. H. (1990): "Structural Model Evaluation and Modification: An Interval Estimation Approach", Multivariate Behavioral Research, No. 25, pp. 173 - 180.

SWIFT, T. (2001): "Trust, reputation and corporate accountability to stakeholders", Bussiness Ethics: A European Review, Vol. 10, No. 1, pp. 16-26.

VARGO, S. L. Y LUSH, R. F. (2004): "Evolving to a New Dominant Logic for Marketing”, Journal of Marketing, Vol. 68 (January), pp. 1 - 17.

VOSS, G. B. Y VOSS Z.G. (2000): "Strategic Orientation and Firm Performance in an Artistic Enviroment”, Journal of Marketing, Vol. 64 (enero), pp. 67 - 83.

WARSHAW, P. R. Y DAVIS, F. D. (1985a): "The accuracy of behavioural intention versus behavioural expectation for predicting behavioural goals", The Journal of Psychology, Vol. 119, No. 6, págs. $599-602$.

WARSHAW, P. R. Y DAVIS, F. D. (1985b): "Disentangling Behavioural Intention and Behavioral Expectation”, Journal of Experimental Social Psichology, Vol. 21, pp. 213 - 228.

WELCH, M. (2006): "Rethinking relationship management. Exploring the dimensions of trust", Journal of Communication Management, Vol. 10, No. 2, pp. 138 - 155.

WESTBROOK Y OLIVER (1991): "The Dimensionality of Consumption Emotion Patterns and Consumer Satisfaction”, Journal of Consumer Research, Vol. 18, junio, pp. 84 - 91.

WOODSIDE, A., FREY, L. Y DALY (1989): “Linking Service Quality, Customer Satisfaction and Behavioral Intention”, Journal of Health Care marketing, Vol. 9 (diciembre), págs. 5 - 7.

ZEITHAML, V .A., BERRY, L. Y PARASURAMAN, A. (1993): "The Nature and Determinants of Customer Expectations of Service”, Journal of the Academy of Marketing Science, Vol. 21 (winter), pp. $1-12$.

ZEITHAML, V. A., BERRY, L. L. Y PARASURAMAN, A. (1996): “The Behavioral consequences of Service Quality", Journal of Marketing, Vol. 60, pp. 31 - 46. 
